Kompetente Antworten innerhalb von 48 Stunden!


\title{
Können Antidiabetika eine Allergie triggern?
}

1 Dr. M. L.: Meine 66-jährige Patientin, niereninsuffizient (Kreatininwert bei 2,2 mg/dl) infolge zweier Teilresektionen beider Nieren (1998 wegen eines Nierenzellkarzinoms, 2012 wegen eines Rezidivs), Hypertonus (unter Medikation normo-, manchmal sogar hypoton), Diabetikerin mit Sensibilitätsstörungen an Händen und Fü-

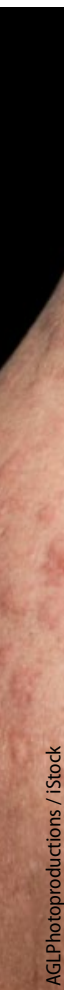

Ben und beginnenden Durchblutungsstörungen an den Zehen, ist mit Sitagliptin bisher gut eingestellt $\left(\mathrm{HbA}_{1 c^{-}}\right.$ Wert: 6,1\%).

Wegen einer Otitis media behandelte ich sie mit Amoxicillin. Eine Penicillinallergie war bis dato nicht bekannt. Am 8. Therapietag, einem Samstag, entwickelte sie zunächst einzelne, später konfluierende, stark juckende Quaddeln, weshalb sie sich in die Notaufnahme eines Krankenhauses begab. Dort nahm man sie stationär auf, weil ihr Kreatininwert auf 2,8 mg/dl angestiegen war. In der Folge klangen die allergischen Reaktionen zunächst nicht ab. Erst nach dem Absetzen von Sitagliptin (und der Umstellung auf Insulin) besserten sich das Hautbild und der Allgemeinzustand der Patientin.

Ist es bekannt, dass Sitagliptin eine Penicillinallergie triggern kann? Betrifft das alle Vertreter dieser Substanzklasse? Auf welches Antidiabetikum sollen wir ausweichen? Und ist die Penicillinallergie reversibel?

MMW-Experte Stiefelhagen: Ihre Schilderungen sprechen für eine Allergie gegen Amoxicillin. Eine solche kann sich auch einmal erst in höherem Lebensalter primär manifestieren. Sie wird auch nicht verschwinden, sodass man in Zukunft ein anderes Antibiotikum verordnen sollte, z. B. ein Cephalo- sporin. Dass Sitagliptin eine solche Allergie triggern könnte, darüber ist mir nichts bekannt, es ist aber auch nicht vollkommen ausgeschlossen. Meines Erachtens könnten Sie die Therapie mit Sitagliptin wieder aufnehmen. Sollte dann die Allergie wieder auftreten, würde ich statt einem Gliptin eine andere antidiabetische Strategie wählen - Insulin oder eine SGLT2-Inhibitor. MMW-Experte FüeßI: Eine allergische Reaktion auf Amoxicillin ist wahrscheinlich. Dass diese durch Sitagliptin ausgelöst worden sein sollte, halte ich für sehr unwahrscheinlich. Allergien gegen Penicillin sind nicht selten und können auch im höheren Lebensalter ohne jede Begleitmedikation auftreten. Der Zusammenhang mit Sitagliptin ist so unwahrscheinlich, dass man es riskieren sollte, diese Therapie fortzusetzen. Auf die Notwendigkeit einer Dosisreduktion bei einer GFR von 22 bis 25 $\mathrm{ml} / \mathrm{min}$ weise ich hin.

Bei einem $\mathrm{HbA}_{1 \mathrm{c}}$ von 6,1\% erhebt sich die Frage, ob die Patientin überhaupt eine medikamentöse Therapie ihres Diabetes mellitus braucht. Wie waren die Blutzuckerwerte vor Sitagliptin? Von einem SGLT2-Inhibitor würde ich bei einer Patientin mit schwerer Niereninsuffizienz abraten. Die Frage nach Insulin stellt sich bei einem so niedrigen $\mathrm{HbA}_{1 \mathrm{c}}$ unter einer Monotherapie mit Sitagliptin wahrscheinlich nicht. 